\title{
Correction to: A vicious circle between insulin resistance and inflammation in nonalcoholic fatty liver disease
}

\author{
Zhonge Chen ${ }^{1 \dagger}$, Rong $\mathrm{Yu}^{2 \dagger}$, Ying Xiong ${ }^{3 \dagger}$, Fangteng $\mathrm{Du}^{3^{*}}$ and Shuishan Zhu ${ }^{3^{*}}$
}

\section{Correction}

Following publication of the original article [1], the corresponding author reported that he had mistyped the first author's unit. The affiliation of Zhonge Chen, "Medical Center of the Graduate School, Nanchang University, China", should be changed to "Department of Gastroenterology, Second Affiliated Hospital, Nanchang University Nanchang, China". All the other authors have agreed to this change. The corrected version should be as follows:

Chen $\mathrm{Z}^{1 *}$, Yu $\mathrm{R}^{2 *}$, Xiong $\mathrm{Y}^{3 * *}$, Du F $\mathrm{F}^{4}$, Zhu $\mathrm{S}^{5}$.

${ }^{1}$ Department of Gastroenterology, Second Affliated Hospital, Nanchang University, Nanchang, China.

${ }^{2}$ Department of Endocrinology, Second Affliated Hospital, Nanchang University, Nanchang, China.

${ }^{3}$ Department of Gastroenterology, Second Affliated Hospital, Nanchang University, No. 1, Minde Road, Nanchang, 330006, China.

${ }^{4}$ Department of Gastroenterology, Second Affliated Hospital, Nanchang University, No. 1, Minde Road, Nanchang, 330006, China. 296978043@qq.com.

${ }^{5}$ Department of Gastroenterology, Second Affliated Hospital, Nanchang University, No. 1, Minde Road, Nanchang, 330006, China.478675878@qq.com.

"Equal contributors.

Address correspondence to

ShuiShan Zhu, Department of Gastroenterology, Second Affliated Hospital, Nanchang University, No. 1, Minde Road, Nanchang 330006, China. E-mail: 478675878@qq.com

Fangteng Du, Department of Gastroenterology, Second Affliated Hospital, Nanchang University, No. 1, Minde Road, Nanchang 330006, China. E-mail: 296978043@qq.com

\begin{abstract}
Author details
'Department of Gastroenterology, Second Affiliated Hospital, Nanchang University Nanchang, Nanchang, China. ${ }^{2}$ Department of Endocrinology, Second Affliated Hospital, Nanchang University, Nanchang, China.

${ }^{3}$ Department of Gastroenterology, Second Affliated Hospital, Nanchang University, No. 1, Minde Road, Nanchang 330006, China.
\end{abstract}

Received: 16 February 2018 Accepted: 16 February 2018

Published online: 23 February 2018

Reference

1. Chen Z, Yu R, Xiong Y, Du F, Zhu S. A vicious circle between insulin resistance and inflammation in nonalcoholic fatty liver. Lipids Health Dis. 2017;16:203. https://doi.org/10.1186/s12944-017-0572-9.

* Correspondence: 296978043@qq.com; 478675878@qq.com

${ }^{\dagger}$ Equal contributors

${ }^{3}$ Department of Gastroenterology, Second Affliated Hospital, Nanchang

University, No. 1, Minde Road, Nanchang 330006, China

Full list of author information is available at the end of the article 